Document downloaded from:

http://hdl.handle.net/10251/147539

This paper must be cited as:

Wang, D.; Wu, T.; Wen, S.; Liu, D.; Xiang, Y.; Zhou, W.; Hassan Mohamed, H.... (2018). Pokemon GO in Melbourne CBD: A case study of the cyber-physical symbiotic social networks. Journal of Computational Science. 26:456-467.

https://doi.org/10.1016/j.jocs.2017.06.009

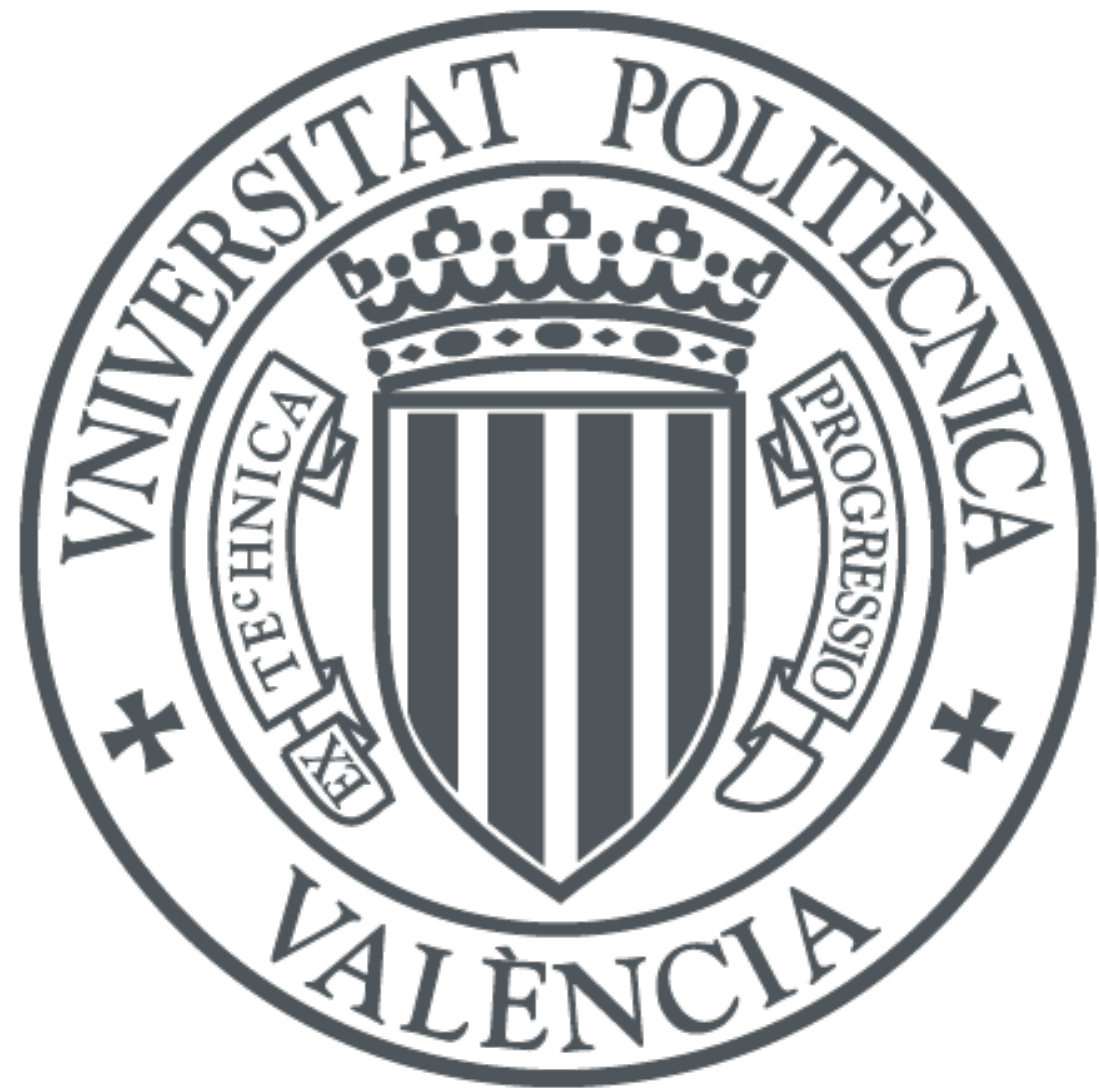

The final publication is available at

https://doi.org/10.1016/j.jocs.2017.06.009

Copyright Elsevier

Additional Information 


\title{
Pokémon GO in Melbourne CBD: A Case Study of the Cyber-Physical Symbiotic Social Networks
}

Derek Wang ${ }^{1,2}$, Tingmin $\mathrm{Wu}^{1}$, Sheng Wen ${ }^{1}$, Donghai Liu ${ }^{1}$, Yang Xiang ${ }^{1}$, Wanlei Zhou ${ }^{1}$, Houcine Hassan ${ }^{3}$, Abdulhameed Alelaiwi ${ }^{4}$

221 Burwood Highway, Burwood, VIC 3125, Australia

\begin{abstract}
The recent popular game, Pokémon GO, created two symbiotic social networks by location-based mobile augmented reality (LMAR) technique. One is in the physical world among players, and another one is in the cyber world among players' avatars. To date, there is no study that has explored the formation of each social network and their symbiosis. In this paper, we carried out a datadriven research on the Pokémon GO game to solve this problem. We accordingly organised the collection of two real datasets. For the first dataset, we designed a questionnaire to collect players' individual behaviours in Pokémon GO, and used maps of Melbourne (Australia) to track and record their usual playing areas. Based on the data that we collected, we modelled the formation of the symbiotic social networks in both physical world (i.e. for players) and cyber world (i.e. for avatars) as well as interactions between players and Pokémon GO elements (i.e. 'bridges' of the two worlds). By investigating the mechanism of network formation, we revealed the relatively weak correlation between the formation processes of the two networks. We further incorporated the real-world

* Corresponding author: Sheng Wen

Email addresses: derek.wang@deakin.edu.au (Derek Wang), tingmin.wu@deakin.edu.au (Tingmin Wu), wesheng@deakin.edu.au (Sheng Wen), wvm@deakin.edu.au (Donghai Liu), yang@deakin.edu.au (Yang Xiang), wanlei@deakin.edu.au (Wanlei Zhou), husein@disca.upv.es (Houcine Hassan), aalelaiwi@ksu.edu.sa (Abdulhameed Alelaiwi)

1 Centre of Cyber Security Research, Deakin University.

2 Data 61, CSIRO, Australia.

3 Polytechnic University of Valencia, Camino de Vera, s/n 46022, Valencia, Spain.

4 King Saud University, Riyadh 11543, Saudi Arabia.
\end{abstract}


pedestrian dataset collected by sensors across Melbourne CBD into the study of their symbiosis. Based on the second dataset, we examined the changes of people's social behaviours in terms of most visited places. The results suggested that the existence of the cyber social network has reciprocally changed the structure of the symbiotic physical social network.

Keywords: Social network, Augmented reality, Pokémon GO.

\section{Introduction}

The movie 'The Matrix' showed us a scene with two connected worlds: physical world and cyber virtual world. Characters can shuttle back and forth between the two worlds and interact with others. Nowadays, this fancy movie

5 scene comes into being due to the emerging Location-based Mobile Augmented Reality (LMAR) techniques [1]. LMAR is changing the form of social networking from multiple aspects. Unlike traditional online social media and offline community, LMAR based social media requires users to physically attend to certain locations for interacting with others. People who interact online using avatars can also derive interactions in the real world when they are physically brought together by LMAR application. Therefore, LMAR has complicated the boundary of social networks, and it also cultivates brand-new forms of social networks. Accordingly, the cyber (virtual) world relationships and the real world connections of people evolve into two symbiotic social networks.

As one part of the symbiotic networks, the cyber social network states the relationships among the online avatars. At the same moment, an offline, locationsensitive physical social network rises among the corresponding users through face-to-face communication, greeting, or more generally, staying in a same area. The emerging mobile game Pokémon GO released by Niantic is a catalyst for symbiotic network formation. The game requires a player to arrive at certain places in order to interact with virtual game content. Players can also battle each other at the in-game facilities called gyms, which are usually pinned on landmark buildings of the real world. The battles between players in the cyber 
space form a cyber social network. On the other hand, since players need to stay in certain areas for playing, their interactions with other players in the real world form an offline physical social network. Hence, the cyber social network and the physical social network symbiotically affect each other.

Previous works about social network mainly focus on studying homogeneous social networks, including the structures, robustness, and user behaviours. The formation and effect of symbiotic social networks has not been deeply examined. Meanwhile, the study of symbiotic network formation can be of great importance to government decision making and society safety. Moreover, the formation of symbiotic social network can be used as a reference and tool in psychological research which studies the formation of social connection and the cognition of human relationship. Potentially, since Pokémon GO and alike applications have great capability to increase physical activity of individuals, this research may assist the analysis of the impact of LMAR on user health.

We carried out a data-driven research to study the symbiotic networks formed by the offline interactions of users and the online connections of the avatars. We studied the properties, user behaviour, and formation of such novel social networks. Accordingly, we used two real datasets in our research. First, we designed a questionnaire to collect Pokémon GO players' individual behaviours in Pokémon GO. Maps of Melbourne were then introduced to track and record players' usual playing areas. Second, we employed pedestrian count data imported from sensors distributed across Melbourne CBD to reflect social behaviours that differ from that of players. Based on the Pokémon dataset, we first built a symbiotic social network of Pokémon GO players. The building process models and simulates the formation of social interaction among the players. Second, we analysed the relationship between the formation of the virtual network and the physical network. Next, we investigated how the social behaviours changed after the symbiotic network emerged. According to our experiment, the popular socialising locations of people have drastically changed after the interference of Pokémon GO. Our work is essential for modelling the information diffusion, the user behaviours, or the impact of AR/VR on human 
interaction in symbiosis social networks.

In this paper, we study the formation, symbiosis and impact of two networks, which are the virtual social network of in-game characters, and the social network of players in the physical world. The paper is organised as follows: methods for collecting and analysing data are presented in Section 2 and 3 . Outcomes of data analysis are exhibited in Section 4. Related works are introduced in Section 5. Finally, this paper is concluded in Section 6.

\section{Dataset 1: Pokémon GO Data ${ }^{5}$}

To investigate the formation of the symbiotic social networks of Pokémon GO player and the symbiosis of the networks, we designed a survey including the related questions.

\subsection{Questionnaire}

We distributed questionnaires to players to collect their playing information (see the details of our questionnaire in Appendix Appendix A). According to the content, the questions can be broadly classified as follows:

Demographics: We asked respondents for their age, title, pokémon master level, pokémon team and interaction preference with other players as the demographics of players. This information was mainly developed to justify the consistency of our survey with existing statistics.

Temporal Information: These kinds of questions are related to time arrangement of players. We recorded the approximate frequency and time that players spent on the game. In addition, we also asked them about their working

5 We submitted and received Deakin's approval for our survey.To protect the privacy of respondents, we avoided asking the personal sensitive details such as name and contact information. In addition, we claimed that all confidential information in the questionnaires would be strictly protected and would not be released. Throughout the survey, we introduced our research team and provided contact information of ours in Deakin University. We also explained our research purpose to participants, some of whom showed interest in our work and expressed strong encouragement. 
or studying time. By these questions, we can conclude the approximate physical social network construction of players. According to the survey results and location data (see details in Subsection 2.2), we can infer the construction of physical and cyber social network for players.

Travelling Distance and Means: To catch more pokémons and challenge gyms, players usually need to travel afar. Therefore, travelling distance and means were investigated as well. This kind of data is expected to extract some preliminary information such as the behaviour comparison among respondents in terms of travelling style.

\subsection{Location Data}

We used printed maps containing annotations of street name, pokéstop and gym to collect areas of playing from participants. The playing location is the key point for deriving the symbiosis of cyber/physical social networks. Considering the fact that asking players to recall the places they travelled to could be difficult to implement online, we decided to use printed map to collect data on street.

We attached two maps with the questionnaire to collect the location information from the Pokémon GO players. The first map is of Melbourne CBD (i.e. small map), and the second map is the zoomed out great Melbourne area (i.e. big map). The big map is $10 \times$ larger than the small map in size. We annotated part of the gyms, pokéstops and pokémons according to the Pokémon GO map application from Google map.

Annotations of virtual content are transposed to the two maps. The distribution of pokéstops on the big map is over-dense, so we excluded pokéstops from the big map. As another fact, gyms are distributed more sparsely and have more significant inner differences than pokéstops. For this reason, compared to the small map, we mainly consider the impact of gyms on the big map. Consequently, the positions of gyms and pokéstops are annotated on the small map, while pokéstops are excluded from the big map 1. 


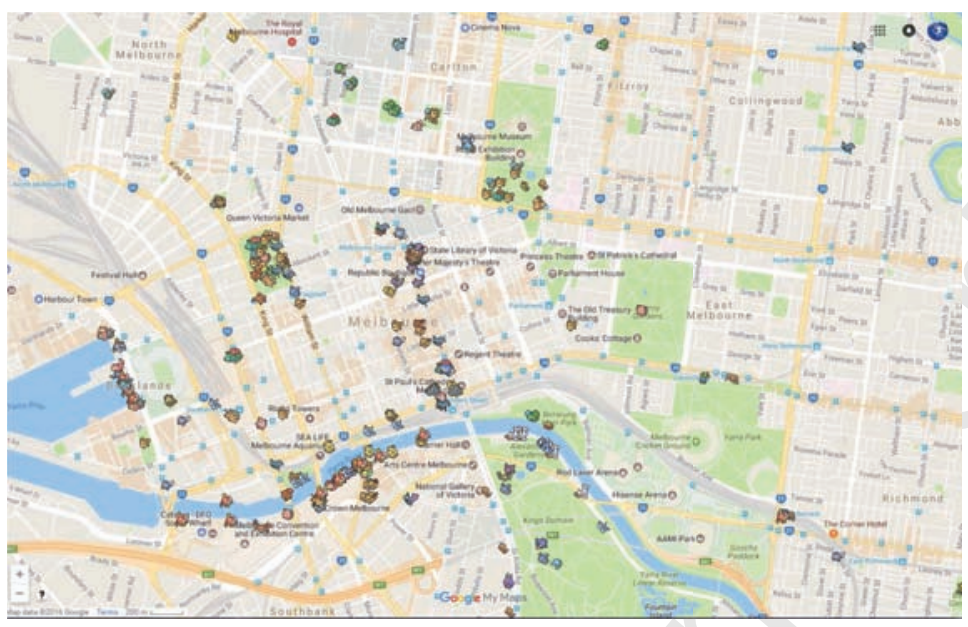

Figure 1: The Melbourne CBD map with pokémon annotated on. Pokémon is an important virtual element in the game. The link between virtual world and physical world is reflected by pokémon-master association.

\subsection{Questionnaire Justification}

In this section, we analyse the information extracted from the questionnaire outcome and compare the result to two Pokémon GO reports.

Preliminarily, we make a statistical study on the responses of the questions. Statistically, the travelling distance for catching pokémon is less than the travelling distance for challenging gym(3). Compared to catching pokémon, players are more likely to challenge gym nearby. From Table 4, we can see that only $37.26 \%$ players had no interaction experience with others while playing games. This presents great opportunities to construct physical social networks among players.

15 The frequency distribution histograms for players in terms of four aspects are shown in Fig. 4. Since there exists null values in the results, we applied the ratio representation instead of the sum in the frequency distribution histogram. If a player had more than one option such as on travelling means, we assumed

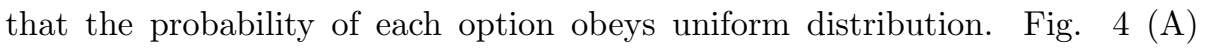
shows the result of how possible a player would challenge gyms in different time periods. We can see that most players prefer to challenge gyms in the evening, 
Table 1: Comparison between Existing Statistics and Our Survey Result

\begin{tabular}{l|r|rrr|rr}
\hline & \multicolumn{2}{c}{ Age } & \multicolumn{2}{c}{ Team } & \multicolumn{2}{c}{ Gender } \\
\hline & $18 \sim 34$ & Yellow & Blue & Red & Male & Female \\
\hline Existing & $78 \%$ & $25 \%$ & $43 \%$ & $32 \%$ & $60 \%$ & $40 \%$ \\
Ours & $93 \%$ & $25 \%$ & $40 \%$ & $35 \%$ & $62 \%$ & $38 \%$ \\
Difference & $15 \%$ & $0 \%$ & $3 \%$ & $3 \%$ & $2 \%$ & $2 \%$ \\
\hline
\end{tabular}

which is out of business time. The occupation is just under $40 \%$ among five breaks. It can be found that less gym challengers are active in the afternoon and night, the percentages of them are no more than half of the ratio towards evening. However, there are just over $10 \%$ players playing in the morning or noon.

We then compared the frequency of travels means selection by players between catching pokémon and challenging gym in Fig. 4(B). It can be concluded that compared to catching pok'emons, players were more likely to use transport vehicles (i.e. car, train, tram and bus) in challenging gyms. In addition, most people preferred to drive their own vehicles to challenge gyms, while they chose not to travel faraway places to catch pokémons.

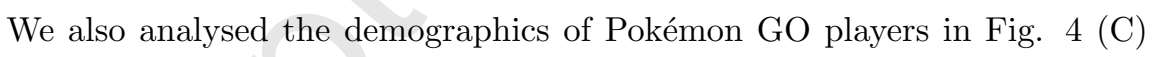
and (D) in terms of age and master level, separately. We can see that the players were mainly from 20 to 30 years old, which accounted for around $80 \%$ of all ages. As for master levels, they were randomly distributed from 5 to 35. Significantly, the number of players at level 22 took a dominant place, the percentage of which were at least twice as any other level. There are rare players whose levels reached to more than 29 , with only a few at 35 .

We further compared our survey result with two existing Pokémon GO statistic analysis reports [2, 3] to justify the rationality of our questionnaire design. It was found that the distribution of our data was corresponding to current statistics in terms of age, team and gender as shown in Table 1. In the table, we removed the null values when each proportion was calculated, so the sum 

are presented in Fig. 2. The coordinates of players were stored in the database. On the other hand, we recorded the density of pokémon, pokéstop and gyms in each grid. The density data were also stored in database. 

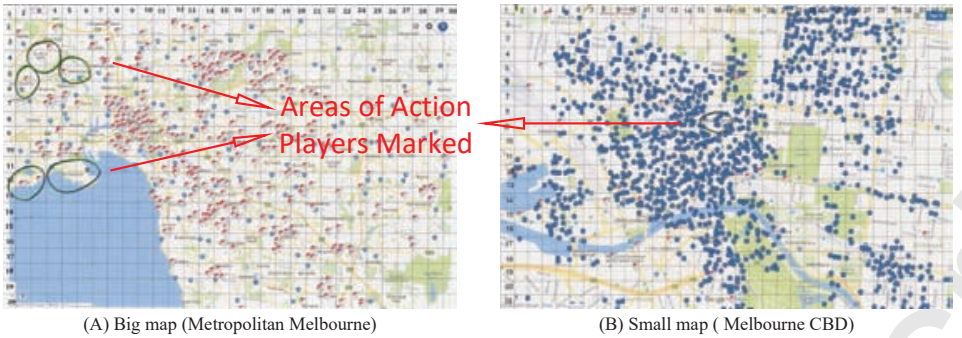

Figure 2: The example of the gridded big map and small map. Within the black circle is the playing area marked by the participant. Blue round dots are pokéstops and the red-white triangles are the gyms.

\section{Dataset 2: Pedestrian Data ${ }^{6}$}

We use the pedestrian dataset to study the impact of the cyber social network to the structure of the physical world in terms of population distribution in the city area. The data is collected by 43 sensors distributed across Melbourne city. These sensors return pedestrian counts of the previous hour monthly. The data has been collected from year 2009 to date. The dataset includes 1,000 sensor records totally, each of which contains the latitude and longitude of the sensor, collecting date/time, the name of the street, and the counted hourly pedestrian number.

\subsection{Computing Pedestrian Density in Grid}

We adopted the pedestrian count value as the hourly density of pedestrian according to the nature of the data. We then computed the pedestrian density in each grid. We transformed the latitude and longitude of each sensor to the position in the mesh grid on the small map. We used Google map API (https://developers.google.com/maps/web-services) to find the latitudes and the longitudes of the mesh grid boundaries. The range of latitude and longitude

6 The pedestrian count data was imported by APIs from Melbourne City Government database (https://data.melbourne.vic.gov.au). The sensors used to collect pedestrian count data were deployed in most popular roads and streets over Melbourne CBD, by government approved agencies. The data was retrieved by sensor logs periodically. 
Table 2: Temporal Statistics: Part I of Questionnaires

\begin{tabular}{lcr}
\hline Time or Frequency & \multicolumn{2}{c}{ Respondents } \\
\hline Time spent on playing & Pokémon GO per week \\
$<0.5$ hour & 10 & $9.62 \%$ \\
0.5 hour $\sim 1$ hour & 14 & $13.46 \%$ \\
1 hour $\sim 2$ hours & 12 & $11.54 \%$ \\
$>2$ hours & 68 & $65.38 \%$ \\
\hline Frequency of playing Pokémon GO & \\
Every hour & 23 & $22.12 \%$ \\
Every day & 55 & $52.88 \%$ \\
One or two days in a week & 17 & $16.35 \%$ \\
Almost forgot it & 9 & $8.65 \%$ \\
\hline
\end{tabular}

Time spent on working or studying per week

$<5$ hours

5 hours $\sim 15$ hours

15 hours $\sim 30$ hours

30 hours $\sim 50$ hours

$>50$ hours

No record

\section{Frequency of challenging gym}

Every day

$15.38 \%$

Few times per week

Few times per month

Never

No record 16

16

22

20

40

4

2

45

17

25

1
$15.38 \%$

$21.15 \%$

$19.23 \%$

$38.46 \%$

$3.85 \%$

$1.92 \%$

of the mesh grid are $[-37.829725,-37.797483]$ and $[144.937239,145.001183]$, respectively. The size of the used mesh grid on the small map is $33 \times 21$. Therefore, we computed the ranges of latitude and longitude of each grid. Given a sensor's location, the sensor will be assigned in the grid whose latitude and longitude 
Table 3: Travelling Distance Statistics: Part II of Questionnaires

\begin{tabular}{llr|lrr}
\hline Distance & \multicolumn{4}{c}{ Respondents } \\
\hline & \multicolumn{4}{c}{ Catching pokémon } & \multicolumn{1}{l}{ Challenging gym } \\
$<1 \mathrm{~km}$ & 17 & $16.35 \%$ & 44 & $42.31 \%$ \\
$1 \mathrm{~km} \sim 5 \mathrm{~km}$ & 36 & $34.62 \%$ & 36 & $34.62 \%$ \\
$5 \mathrm{~km} \sim 10 \mathrm{~km}$ & 24 & $23.08 \%$ & 14 & $13.46 \%$ \\
$10 \mathrm{~km} \sim 20 \mathrm{~km}$ & 13 & $12.50 \%$ & 2 & $1.92 \%$ \\
$>20 \mathrm{~km}$ & 14 & $13.46 \%$ & 5 & $4.81 \%$ \\
No record & 0 & $0.00 \%$ & 3 & $2.88 \%$ \\
\hline
\end{tabular}

range covers the sensor's location. We could therefore get the positions of the sensors in the mesh grid. The pedestrian density in a grid thus equals the pedestrian count in the grid. For a grid containing multiple sensors, the density is set as the average pedestrian number from all sensors in the grid.

\subsection{Completing Pedestrian Density}

Since the pedestrian density of the grid in which there is no sensor was unknown, we first completed the pedestrian density in this section. We inferred the density of pedestrian in each grid based on a regression model. We compared three regression models, namely radial basis kernel support vector regression (SVR) [4], kernel ridge regression (KR) [5], and linear regression (LR) [6].

Initially, we extracted features for the regression models. Suppose we have a set of grids $G_{\text {all }}$. $G_{S} \subseteq G_{\text {all }}$ are the set of grids in which there are sensors. The set of grids in which the pedestrian density will be estimated therefore is $G_{E}=G_{\text {all }}-G_{S}$. We compared three different metrics and adopted one as the feature for regression. Since the distribution of people highly related to spatial information, the three evaluated metrics utilise spatial distance and pedestrian count value.

As the first metric, for each grid $g_{k} \in G_{\text {all }}$, we computed the average 
Table 4: Demographics Statistics: Part III of Questionnaires

\begin{tabular}{lrr}
\hline Demographics & Respondents \\
\hline Title & 63 & $60.58 \%$ \\
Mr & 6 & $5.77 \%$ \\
Mrs & 33 & $31.73 \%$ \\
Miss & 0 & $0.00 \%$ \\
Dr & 0 & $0.00 \%$ \\
Other & 2 & $1.92 \%$ \\
No record & & \\
\hline Team & 40 & $38.46 \%$ \\
Blue & 35 & $33.65 \%$ \\
Red & 25 & $24.04 \%$ \\
Yellow & 3 & $2.88 \%$ \\
No team & 1 & $0.96 \%$ \\
No record & & \\
\hline
\end{tabular}

Interaction preference

\begin{tabular}{lrr} 
Greeting & 37.75 & $36.30 \%$ \\
Grouping & 6.25 & $6.01 \%$ \\
Friending & 19.25 & $18.51 \%$ \\
No interaction & 38.75 & $37.26 \%$ \\
No record & 2 & $1.92 \%$ \\
\hline
\end{tabular}

Note: No record: the player failed to answer the question. When asked about interaction preference, some players had more than one choices. Therefore, for those players, we divided 1 by the number of choices, and added each division to the cumulative value of respondents.

distance-weighted density coefficient $C$ :

$$
C\left(g_{k}\right)=\frac{\sum_{g_{j} \in G_{S}} \frac{n_{g_{j}}}{\operatorname{Dist}\left(g_{k}, g_{j}\right)^{2}}}{N}
$$

wherein $n_{g_{j}}$ is the pedestrian count in grid $g_{j} . N$ is the number of grids in $G_{S}$. 
$\operatorname{Dist}\left(g_{k}, g_{j}\right)$ is the Euclidean distance between grid $g_{k}$ and $g_{j}$. Second, for each $g_{k} \in G_{a l l}$, we recorded the pedestrian count $N C\left(g_{k}\right)$ in the nearest grid of $g_{k}$ in $G_{S}:$

$$
N C\left(g_{k}\right)=n_{g_{j}}, \underset{g_{j}}{\arg \min } \operatorname{Dist}\left(g_{k}, g_{j}\right)
$$

Finally, we used the distance from $g_{k}$ to $g_{j}, \arg \max _{g_{j} \in G_{S}} n_{g_{j}}$ as the third metric, $D\left(g_{k}\right)$. We built a training sample set based on the features. There are 37 grids in $G_{S}$. For each $g_{j} \in G_{S}$, we computed $C\left(g_{j}\right), N C\left(g_{j}\right)$ and $D\left(g_{j}\right)$ as features, which is labelled by the pedestrian count $n_{g_{j}}$ in $g_{j}$. Thus 37 training samples were built in this manner.

To achieve better performance, we employed cross validation to evaluate regression performance and chose the best model. We split $30 \%$ of the total training samples as testing samples in cross validation. Then, we evaluated regression performance in terms of mean average error (MAE), mean average percentage error (MAPE), explained variance score (EVS), and $R^{2}$ score. We also tuned the features used in regression to improve the regression performance. By solely using $C\left(g_{k}\right)$, the regression models gained the best performance. Among the regression models, SVR using radial basis kernel performed as the best regression model on all metrics (Fig 31). Thus we adopted SVR using radial basis kernel to infer pedestrian density for each $g_{e} \in G_{E}$. The inferred pedestrian density is utilised in Section IV C.

\section{Results}

\subsection{Preliminary Analysis on Questionnaire and Location}

Table 5: PCC between player and virtual content

\begin{tabular}{ll} 
Player/Gym & 0.069 \\
Player/Pokémon & 0.385 \\
Player/Pokéstop & 0.480 \\
\hline
\end{tabular}

In this section, we present the statistics of the survey participants and their playing behaviours according to the questionnaires. Participants are anonymised 


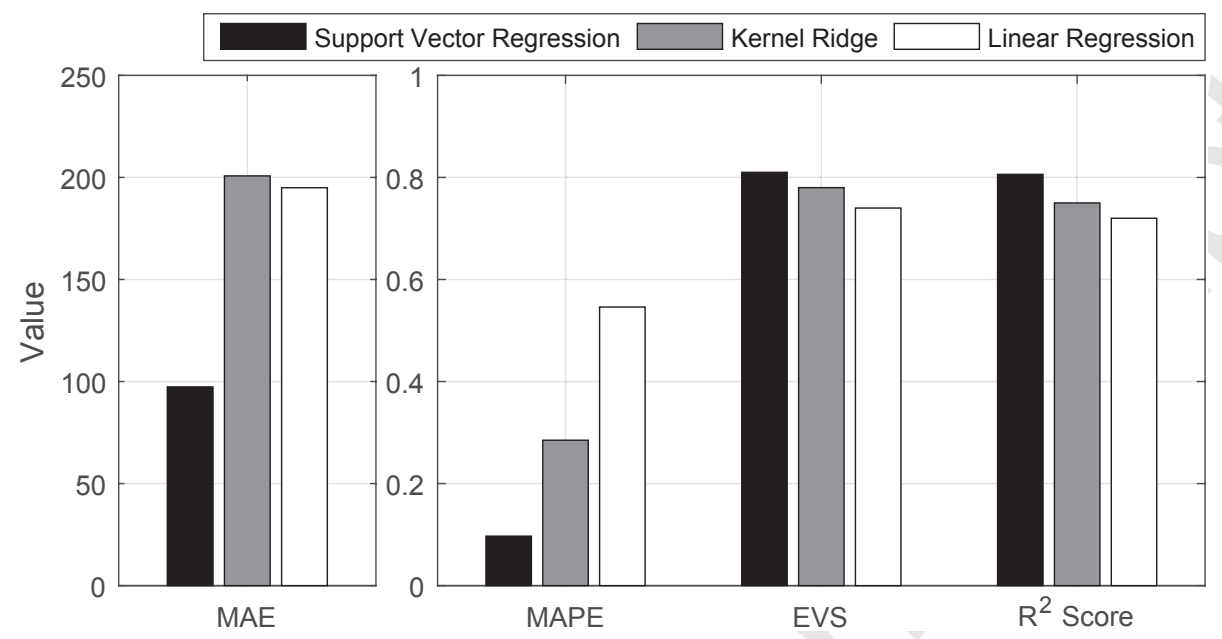

Figure 3: The performance comparison of SVR, Kernel Ridge, and Linear Regression. SVR achieves best performance in terms of MAE, MAPE, EVS and $R^{2}$ score.

for privacy considerations. As shown in Table 2. 75\% of the 104 respondents played Pokémon GO every hour or every day, while there were only around $15.38 \%$ of the players challenged gym at the same frequency. It is deduced that players were much less frequent in challenging gym than catching pokémon.

Next, we analysed the characteristics of the obtained data in terms of player location distribution, gym distribution, pokéstop distribution, pokémon distribution on the big and small map. Based on the collected location data with the mesh grid, we plotted the heat map of distributions in Fig. 5. For each grid, we accumulated the number of player whose playing area includes the grid. The accumulated value is the number of player in this grid. In this manner, we got the relative density distribution of players on the whole mesh grid. The relative density of virtual content on the mesh grid is counted in the same way. It can be observed from Fig. [5 that players are mainly distributed in the CBD area on the map of greater Melbourne. As for the locations on the zoomed in small map, players are gathered in the city central and south bank areas. We then analysed the correlations of the distributions. 


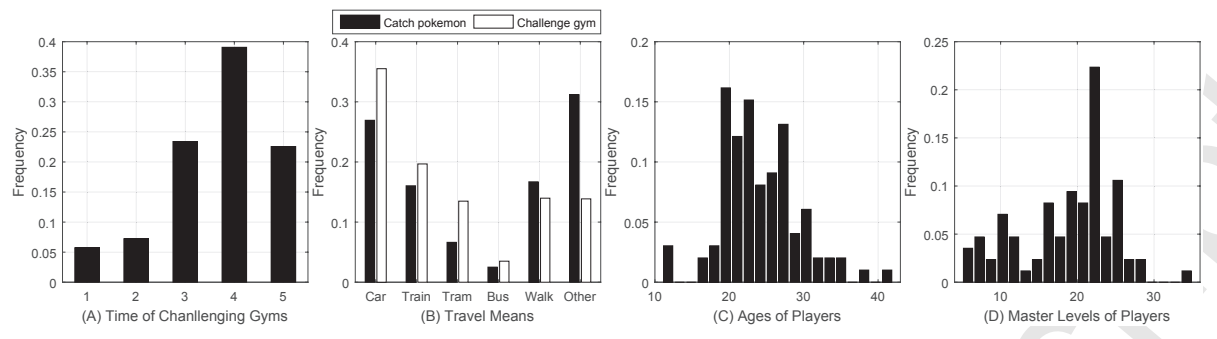

Figure 4: Our preliminary statistical analysis of the questionnaires are based on the followed frequency distributions: (A) Time of players challenging gyms (note: '1': Morning, '2':Noon, '3': Afternoon, '4': Evening, and '5': Night.), (B) Travel means selected in catching pokémon and challenging gyms (note: 'Other' represents the players who never travelled far away.), (C) Ages of players, and (D) Master levels of players.

\subsection{Formation and Symbiosis of Symbiotic Social Networks}

\subsubsection{Physical Social Network: Human Interaction in Real World}

The interactions among players are analysed in this section. We assume that two players staying in the same grid at the same time of a day have the potential to physically meet and interact with each other in the real world. We then estimate the probability of meeting, which indicates the likelihood that social activity occurs between the two players.

We first defined $P_{u, v}$ as the probability that two players $u$ and $v$ come across each other in the physical world. In other words, $P$ stands for the formation likelihood of the interaction between a new player and other existing players. As an intuitive assumption, we assume that: 1) $P(u, v)$ decreases with increasing distance between $u$ and $v$; 2) $P(u, v)$ decreases with increasing time interval between the timing when $u$ or $v$ plays the game. Second, we define $P_{u, v}$ as 1 when $u$ and $v$ stay in the same grid in the same hour of a day. According to the previous research, the timing of many human activities follows Poisson distribution [7]. Furthermore, as a statistical property of Poisson processes, the time intervals between consecutive events follow an exponential distribution, while distributions obeying power-law are also observed [8]. According to the property held by the time interval, in this research, we estimate the probability $P(u, v)$ using the following model. The model serves as a prompt for the utilisation of 

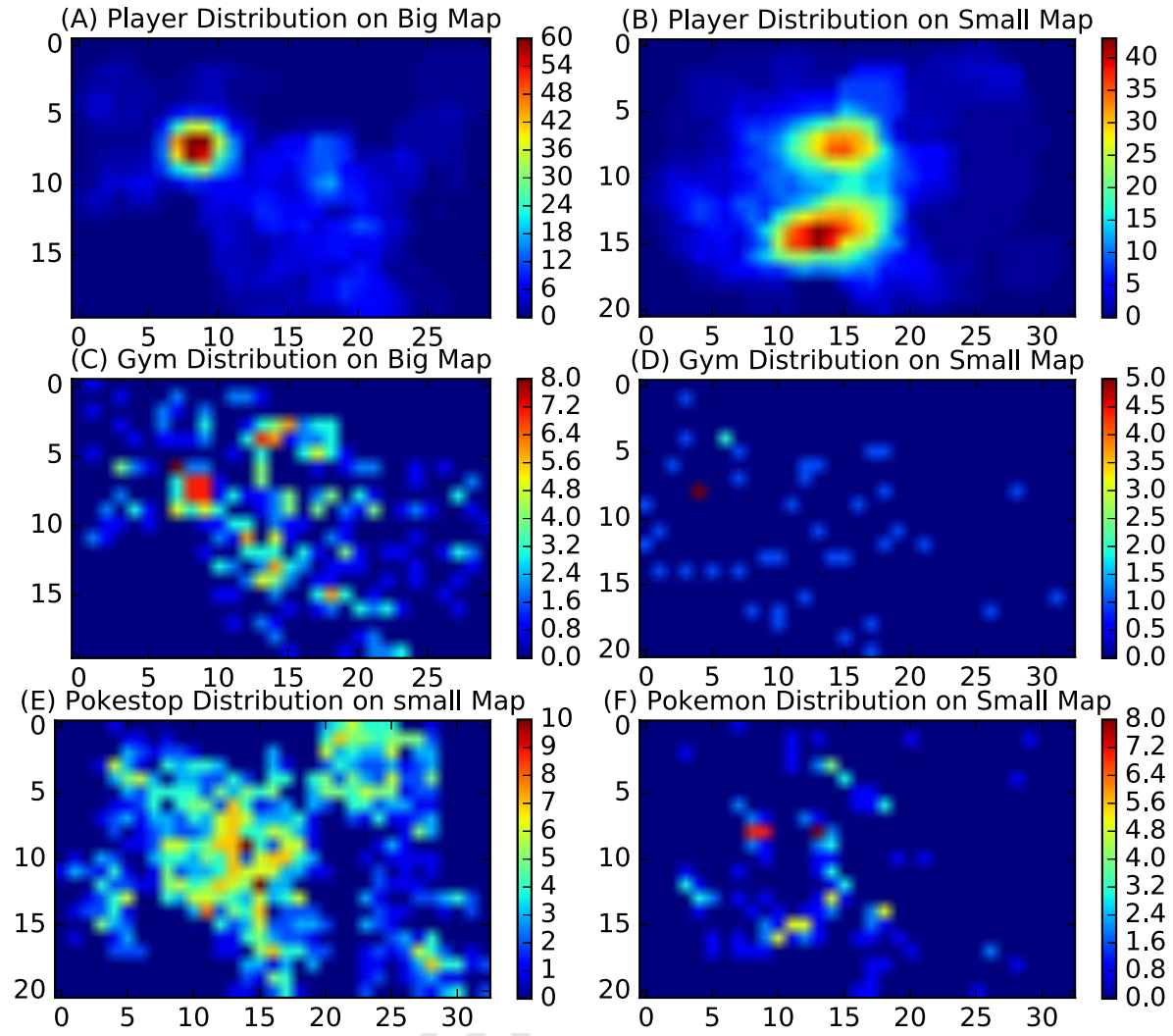

Figure 5: The distribution heat maps of player, gym, pokéstop, and pokémon in Melbourne.

this dataset. Extension and better model can be proposed in the future.

$$
\left\{\begin{array}{l}
P(u, v)=\alpha \cdot e^{-\alpha \cdot \Delta t_{u, v}} \text { or } P(u, v)=\alpha \cdot \Delta t_{u, v}^{-1-\alpha} \\
\alpha=\frac{1}{e^{\Delta S_{u, v}}}
\end{array}\right.
$$

wherein $\alpha$ is the penalty on the probability based on the distance between the locations of the players (Fig. 6(B)). $\Delta S_{u, v}$ denotes the average Euclidean

distance between $u$ and $v . \Delta S_{u, v}$ is computed as: 


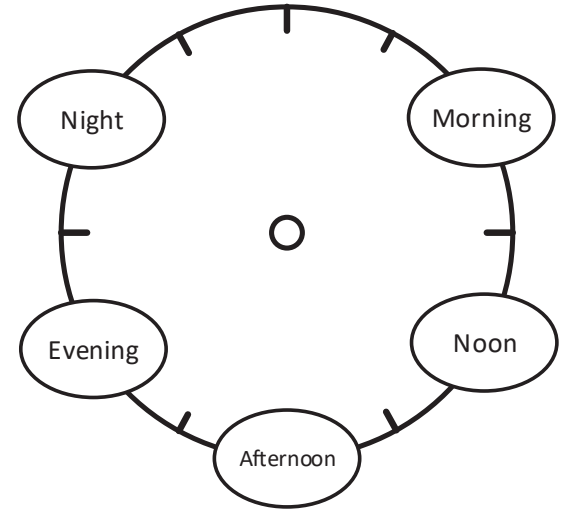

(A)

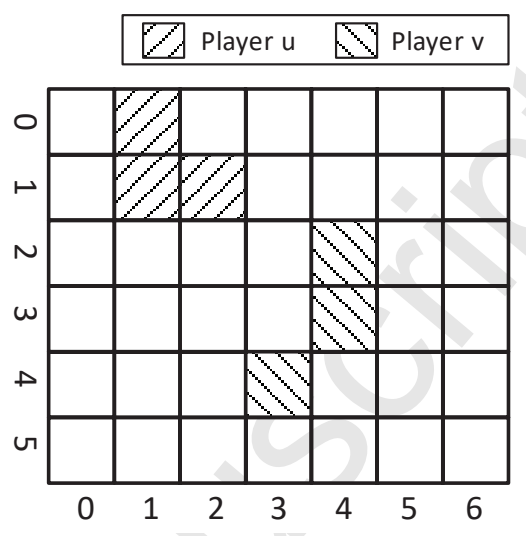

(B)

Figure 6: (A) The intervals between different time of a day. The interval between night and morning is 8 hours, and others are 4 hours. (B) The locations of user $u$ and $v$ in the mesh grid.

$$
\Delta S_{u, v}=\sum_{i \in A(u), j \in A(v)} \operatorname{dist}(i, j)
$$

herein $i, j$ are the coordinates of the grids that are from the active areas of player $u$ and $v$, respectively. $\operatorname{dist}(i, j)$ is the Euclidean distance between $i$ and j. $\Delta t_{u, v}$ is the time interval (Fig. 6(A)). Specifically:

$$
\Delta t_{u, v}= \begin{cases}8 & \text { between night and morning } \\ 4 & \text { Otherwise }\end{cases}
$$

We calculated the defined $P$ of all the surveyed players. By setting a cutoff of $P$, the network in which $P$ between any player pair exceeds the cutoff is selected. A physical social network conditioned by the cutoff selection is therefore built. We swept the cutoff from 0.1 to 0.9 in steps of 0.1 . We considered the generated network as non-trivial for studying if the network has average node degree larger than 1 . Therefore, we set the cutoff as 0.2 in this paper to obtain 
(A)

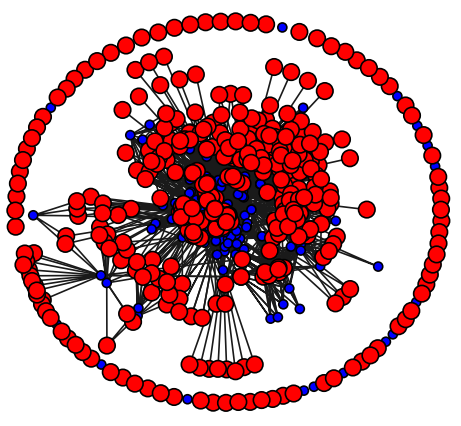

(C)

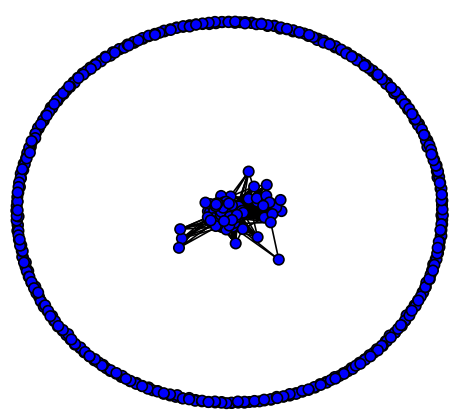

(B)

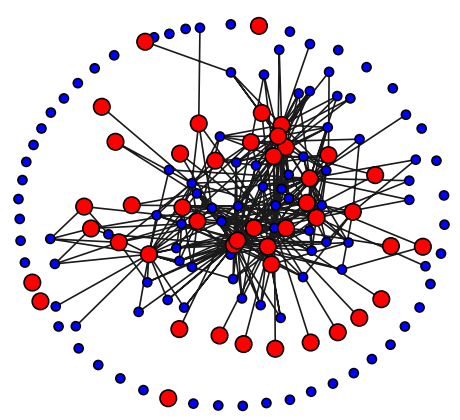

(D)

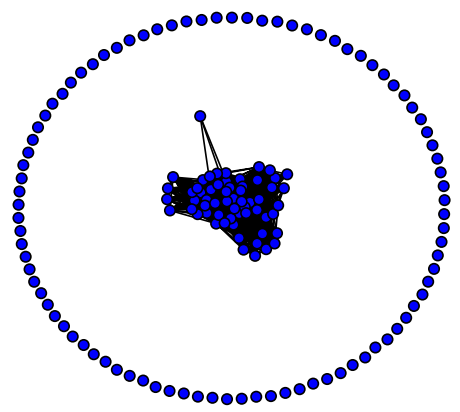

Figure 7: The interactions between the avatars of the 104 players and the gyms on the maps. (A) The virtual network of avatars, including gyms, on big map. (B) The virtual network of avatars, including gyms, on small map. Red nodes are gyms and blue nodes are avatars. The edge standing for the existence of interaction links the character and the gym if the gym is in the location of the player to whom the character belongs. (C) The joint network of avatars and players on big map. (D) The joint network of avatars and players on small map. There is an edge connecting two avatars if their players share the same gyms in their playing areas to form the virtual interaction. Isolated nodes are also plotted to show the avatars without interaction with others.

the non-trivial network. The value can be tuned to acquire network of different scales. 


\subsubsection{Virtual Social Network: Avatars in Virtual World}

In this section, the analysis about the interactions among avatars (e.g. pokémon masters) is presented. The gym is the sole venue in the game for the avatars to interact. Once players win the gym battles, they become leaders of the gyms and their pokémons will be selected as gym guardians. Then other players' pokémons can interact with the defenders even when the gym leaders are not geographically close to the gym. From another perspective, avatars in the game derive interaction at the gyms. Therefore, the interaction activity through challenging gym constructs a virtual social network among pokémons.

Firstly, we obtain the virtual interaction network of gyms and avatars based on the assumption that once the playing area of a player has a gym in it, the interaction between the avatar of the player and the gym is existing. The virtual network is plotted in Fig 7 (A) and (B). In fact, the edge and gym nodes in the network are dynamic.

Then, we calculated the interaction probability of two arbitrary avatars in the pokémon world. In the world, each avatar was mapped as the pokémon belonging to each player. We assumed that a players challenges each gym in their area at the same probability. Given two avatars $u_{v}$ and $v_{v}$ which are corresponding to two players, their meeting probability in virtual world can be expressed as below:

$$
P^{\prime}\left(u_{v}, v_{v}\right)=n\left(u_{v}, v_{v}\right) /\left(n\left(u_{v}\right) * n\left(v_{v}\right)\right)
$$

wherein $n\left(u_{v}\right)$ and $n\left(v_{v}\right)$ represent the number of gyms in the area of $u$ and $v$ respectively, and $n\left(u_{v}, v_{v}\right)$ means the number of gyms in the common area of $u$ and $v$. In the cyber social network, there is an edge connecting two avatars

300 if their players share the same gyms in their playing areas to form the virtual interaction. Differing from the $P$ of physical interaction probability, we used $P^{\prime}$ as the weights of edges in the network. 


\subsubsection{Symbiosis: Player and Virtual Content} in which more players gather. According to Fig. 8 and Fig. 5, we can observe that the density of players and the density of virtual content show positive cor- 
relations. However, the gradients of the densities are highly distinguishing.

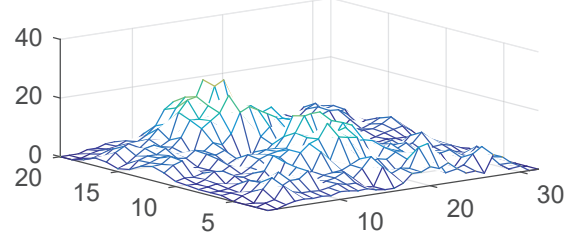

(A) Player to Stop on Small Map

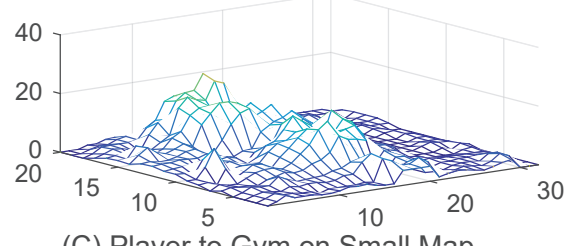

(C) Player to Gym on Small Map

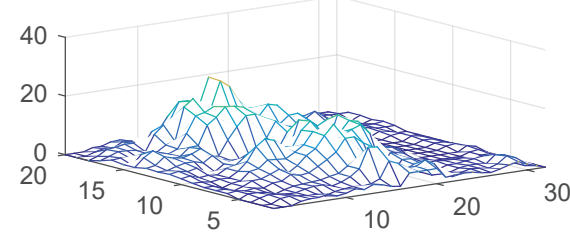

(B) Player to Pokemon on Small Map

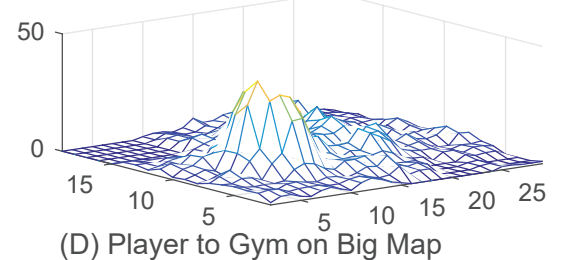

Figure 8: The height represents the the difference in the gradient of player distribution and the gradient of virtual world distributions of pokémons, pokéstops and gyms. Fig. A, B and $\mathrm{C}$ are based on distributions on small map, and Fig. D is based on distributions on big map.
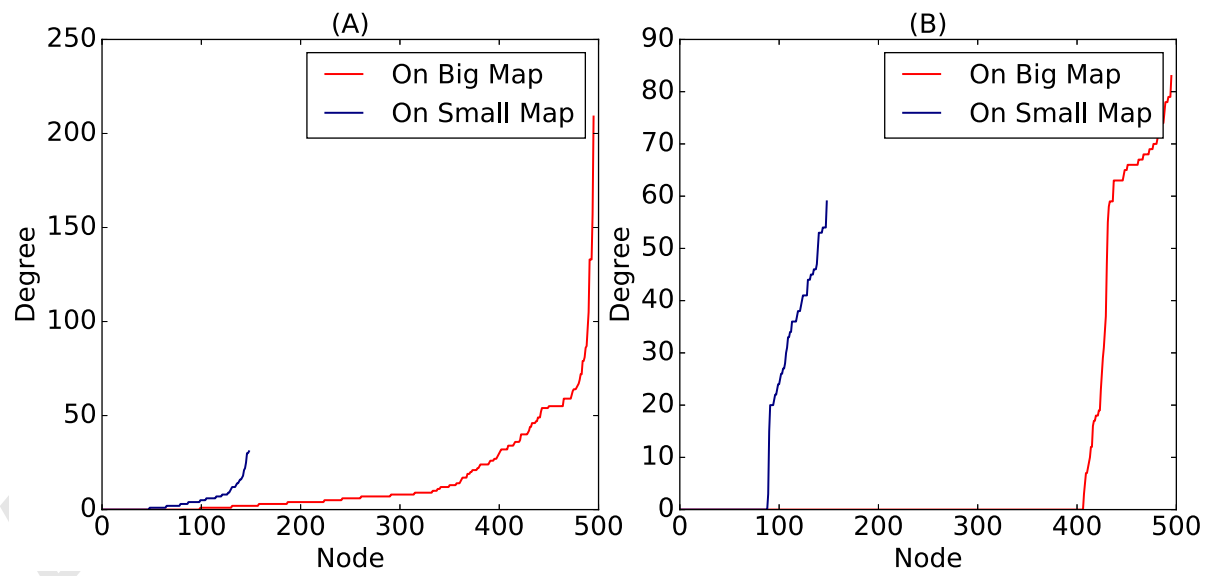

Figure 9: (A) The distribution of node degree in the cyber social network. The degrees demonstrate a power-law alike distribution. (B) The joint network of two symbiotic networks of avatars and players. The degree distribution differs from the power-law distribution. 


\subsection{Impact of Symbiotic Networks}

We investigated the impact of the symbiosis on cyber social network and the physical activity network in this section.

\subsubsection{Relationship between Formation Process} players could not sufficiently reflect the physical behaviours conversely due to the low PCC value in both scenarios.

Table 6: PCC between interaction probability among physical players and avatars

\begin{tabular}{ccc} 
& Time considered & Time eliminated \\
\hline Big map & 0.3046 & 0.3220 \\
Small map & 0.0784 & 0.0848 \\
\hline
\end{tabular}




\subsubsection{Change on Social Network and Social Behaviour}

In this section, we first analysed the change on degree distribution of nodes in the social network. We then investigated how the location based mobile AR app can affect people's physical social behaviour. We compared the socialising hot-spot of Pokémon GO players and normal pedestrians in Melbourne CBD area.

We first analysed the topological characteristics of the cyber social network formed by the interactions among avatars. The degree distribution of the networks are illustrated in Fig. 9. It can be observed that the degrees of the network formed by gyms and avatars roughly obeys the power-law distribution, which is typical for online social networks. Secondly, we joined the cyber social network formed by avatars and the physical social network formed by people on associated players and their avatars. The joint networks are plotted in Fig[ (C) and (D). The degree distribution of the joint network demonstrates a nonpower-law distribution. The degree changes more rapidly than the power-law curve.

Second, we looked into the change on the physical social behaviour of people, in particular, the popular socialising venue of people. We compared the pedestrian density inferred in Section II in Fig. 10. We considered the following 5 groups of sensor data: 1) The average count value of all time; 2) The average count between 00:00 to 12:00 of all time; 3) The average count between 12:00 to 24:00 of all time; 4) The average count between Thursday to Sunday of all time; 5) The average count within every August since 2009. Since we collected player location data in August, from Thursday to Sunday in the evening/night, the setting of these rules helps us to unify the conditions for comparison.For each group of data, we correspondingly trained SVR and inferred the density of all grids without sensors.

We compared the social hot-spots of the non-player pedestrians and the players. The highly populated areas usually suggest the existence of more social activities. Therefore, by comparing the density of people, we intend to reflect 

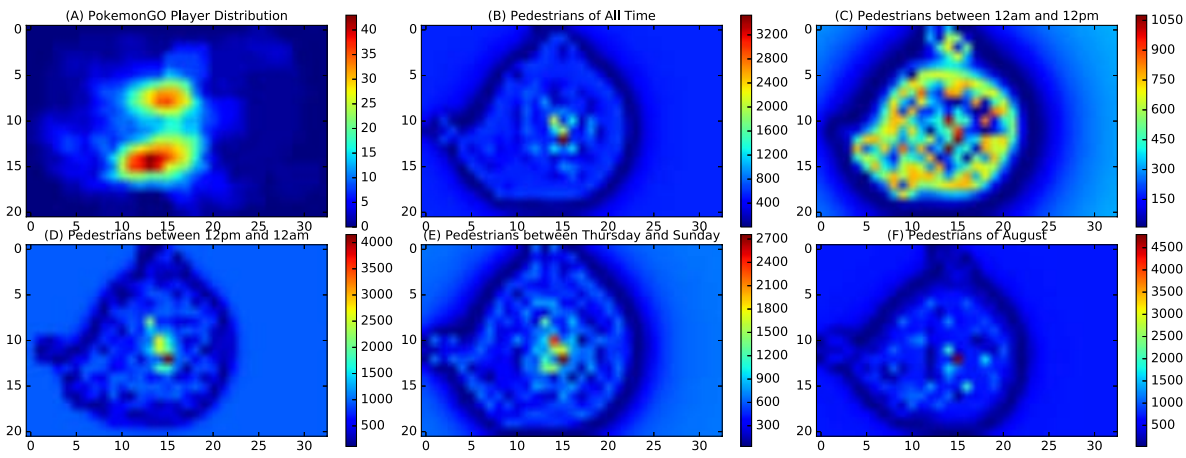

Figure 10: Comparison of the relative densities of Pokémon GO players and pedestrians on the small map. The colour bar at the right side of each plot indicates the density of people. The distributions show differences in the locations of player and non-player.

the area with more social activities. According to Fig. 10, the social hot-spot of pedestrians is around $(15,13)$ while the hot-spots of Pokémon GO players are located in grid $(13,15)$ and $(15,8)$, where have most of pokémons gathered. Players shift to the popular locations under the effect of the AR based app according to the aggregation of virtual content.

\section{Related Work}

The characteristics and interactions of traditional online/offline social networks have been exclusively analysed. On the other hand, the perception regarding location based mobile AR applications is evolving. It is of interest to understand how AR can affect social behaviour of users, and what the characteristics of the social network would then have. We provide a brief review of the research about social network characteristics and location based AR. As the latest phenomenal LMAR application, Pokémon GO has also been introduced in terms of the impact it brings and the perception it receives.

Analysis on Single Social Networks The topological structures of social networks and the user behaviours in the networks have been extensively studied.

Social network could be broadly categorised into two kinds, namely static social network and dynamic social network. Currently, the social networks are mainly 
dynamic. For example, the online social network (OSN) is one representative type of social networks. The studies around OSNs (e.g. Twitter, Facebook, Flickr, and Yahoo! 360 etc.) have revealed the features of impacting events diffusing in the network, and clues behind OSN user behaviours [9, 10]. A study conducted by Kumar et al. analysed the evolution and dynamics of the social network. Most of the studies address the topological feature (e.g. degree distribution, density, cluster coefficient, connectivity etc.) of the networks [11]. Second, studies on social networks from the socio-technical and user behavioural perspectives were conducted [12, 13, 14]. Also, the attributes of social network were also studied. For example, Heravi et al. studied the privacy calculus decision making processes in OSN [15], and David et al. found that a static network is more helpful in constructing stronger cooperation than traditional population distribution [16]. Apart from the single social network, there are also few studies about the symbiotic social network. Yagan et al. studied the information diffusion in conjoining social networks and revealed that information transmission speed and scale were remarkably larger in the conjoining online and physical social network than in the single social network [17]. However, though a few of the current works catch a glimpse of the symbiotic social network, the analysis on formation, feature, and user behaviour of LMAR based symbiotic social network was not provided.

Interconnected Networks To better understand the physical interactions for individuals, complex networking models are generally employed. In fact, there are many sub interconnected networks in the real-world scenarios. For instance, Dickison et al. and Wang et al. developed the epidemics effect through interconnected networks [18, 19]. In their studies, the impact on epidemic broadcasting was analysed according to different connection strength of two mutually interdependent networks. Likewise, Radicchi experimentally explored the spectral features in the networks with different dynamic and topological properties such as degree distributions and connection distinction between intra-connected networks and interconnected networks [20]. In addition, the attribute robustness was also studied by researchers. For example, Parhami proved that swapped 
networks were significantly robust and they have relatively small degrees of nodes [21]. Radicchi and Arenas also proposed that although there were sufficient distinct attributes such as robustness for the interconnected networks, abrupt transition would also occur inwards [22]. By contrast, our study about Pokémon GO mainly focuses on the formation of the symbiotic networks, where each connection is weighted numerically.

Location based Augmented Reality Differing from previous studies that focused on the technical details of AR components [23], research on mobile AR usage concentrates on the acceptance, user experience, and perceptions of mobile AR functionality [24]. Alternatively, researchers conducted experiments to test how mobile AR applications can be useful for certain applications such as navigation [25]. Investigation on the usage of Layar, which is a widely installed mobile AR application, exhibits the formation of social practises around mobile AR, and how emerging media may complicate the practices, experiences, and relationships in the spatial landscape[26]. Similar to Pokémon GO, Layar can overlay points of interest, annotations, or pictures on camera input of the realworld scene based on global positioning system. The development of an LMAR application named CityViewAR was presented as well [27, 28]. The study mainly focused on the design and the implementation of the application. Though studies about mobile AR related applications are continuously presented, there is no research modelling the social behaviours of users under LMAR technology. There is also an absence of study that analyses the relations between physical social networks and virtual contents from the LMAR application.

Impact of Pokémon GO The impact of Pokémon GO on player's physical activity has recently been studied [29]. The results of the study suggest that Pokémon GO can significantly increase the daily physical activity of players. Likewise, it was found that social comparison in online social networks could 465 effectively activate individuals to do physical activity [19, 30]. The impact brought by Pokémon GO on traffic, personal security and privacy has attracted attention from government in some countries [31, 32, 33]. Current studies or perceptions about Pokémon GO are mainly focusing on the pros and the cons of 
Pokémon GO on health and psychology. There is neither comprehensive analysis behaviours, nor study from the social network perspective.

\section{Conclusion}

In this paper, we analysed the symbiosis and characteristics of symbiotic social networks among users of a location based augmented reality application.

475 For studying symbiotic social network formation, we collected a Pokémon GO player dataset which contains the player demographics, playing behaviours, and playing locations. By incorporating real world pedestrian count data, we studied the change on social behaviour brought by symbiotic social network in terms of spatial hot-spot deriving social activity. For the first time, this paper offers a clue for the study of symbiotic social networks. This study provides a series of results from real-world data, which will contribute to the future research on large-scale symbiotic social networks. The research has its limit. Through survey, the accuracy of the collected player location data is varying. Even though we required the respondents to mark their location as accurate as possible, cial capital and influence across traditional commerce, e-commerce, and social 
commerce platforms. Social support and companionship deriving from social networks have also been shown to impact profoundly on mental health [34]; however, studies have predominately focused on the effects of either physical social networks (e.g. family, romantic relationships, friends) or virtual social networks (e.g. social media). A greater understanding of the formation and operation of cyber-physical symbiotic social networks could provide insight into the specific effects, whether positive or otherwise, of LMAR technology on mental health. Further, cyber-physical symbiotic social networks may provide different levels or types of social support compared with purely physical or virtual/cyber social networks.

\section{Appendix A. Questionnaire}

This is the questionnaire that was handed to Pokémon GO players in the Melbourne city.

Appendix A.1. Demographics

1. What is your title? [Mr, Mrs, Miss, Dr, Other]

2. What is your age?

3. What is your Pokémon master level?

4. Which Team did you select? [Blue, Red, Yellow, No team]

5. Do you interact with other players in the real world? [Greet them, Group with them, Friend them, No interaction]

\section{Appendix A.2. Temporal}

1. Approximately, how much time do you spend on playing Pokémon GO per week? [Less than half an hour, Half an hour $\sim 1$ hour, 1 hour $\sim 2$ hours, More than 2 hours]

2. How frequent do you play Pokémon GO? [Check it every hour, Play it casually every day, Play it a day or two in a week, Almost forgot it] 
3. How much time do you spend on working or studying per week? [Less than 5 hours, 5 hours $\sim 15$ hours, 15 hours $\sim 30$ hours, 30 hours $\sim 50$ hours, More than 50 hours]

4. How frequent do you go to challenge gym? [Every day, Few times per week, Few times per month, Never]

5. What time in a day are you likely going to challenge gym? [Morning, Noon, Afternoon, Evening, Night]

\section{Appendix A.3. Travel Distance and Means}

1. What transport do you take when you travel to faraway places for catching rare pokémon? (You may choose multiple answers) [Private vehicle, Train, Tram, Bus, On foot, I don't travel to faraway places]

2. Within how long of travelling distance do you think is acceptable for catching rare pok'emon? [Less than $1 \mathrm{~km}, 1 \mathrm{~km} \sim 5 \mathrm{~km}, 5 \mathrm{~km} \sim 10 \mathrm{~km}, 10 \mathrm{~km}$ $\sim 20 \mathrm{~km}$, More than $20 \mathrm{~km}$ ]

3. What transport do you take when you travel to faraway places for challenging gyms? (You may choose multiple answers) [Private vehicle (driving or passenging), Train, Tram, Bus, On foot, I don’t travel to faraway places]

4. Within how long of travelling distance do you think is acceptable for catching rare challenging gyms? [Less than $1 \mathrm{~km}, 1 \mathrm{~km} \sim 5 \mathrm{~km}, 5 \mathrm{~km} \sim 10$ $\mathrm{km}, 10 \mathrm{~km} \sim 20 \mathrm{~km}$, More than $20 \mathrm{~km}$ ]

\section{Appendix B. Acknowledgement}

This research is partially supported by the Australian Research Council projects DP150103732, DP140103649, and LP140100816. The authors extend their appreciation to the International Scientific Partnership Program (ISPP) at King Saud University, Riyadh, Saudi Arabia for funding this work through the project No. ISPP\#0069. 


\section{Appendix C. References}

[1] P. Geiger, M. Schickler, R. Pryss, J. Schobel, M. Reichert, Locationbased mobile augmented reality applications: Challenges, examples, lessons learned.

[2] 75 incredible pokemon go statistics (november 2016), http://expandedramblings.com/index.php/pokemon-go-statistics/.

[3] 121 pokmon go statistics for family fun (november 2016), http://www.rawhide.org/blog/infographics/pokemon-go-statistics/.

[4] A. J. Smola, B. Schölkopf, A tutorial on support vector regression, Statistics and computing 14 (3) (2004) 199-222.

[5] S. An, W. Liu, S. Venkatesh, Fast cross-validation algorithms for least squares support vector machine and kernel ridge regression, Pattern Recognition 40 (8) (2007) 2154-2162.

[6] J. Neter, M. H. Kutner, C. J. Nachtsheim, W. Wasserman, Applied linear statistical models, Vol. 4, Irwin Chicago, 1996.

[7] A.-L. Barabasi, The origin of bursts and heavy tails in human dynamics, Nature 435 (7039) (2005) 207-211.

[8] R. D. Malmgren, D. B. Stouffer, A. E. Motter, L. A. Amaral, A poissonian explanation for heavy tails in e-mail communication, Proceedings of the National Academy of Sciences 105 (47) (2008) 18153-18158.

[9] H. Kwak, C. Lee, H. Park, S. Moon, What is twitter, a social network or a news media? in: Proceedings of the 19th International Conference on World Wide Web, WWW '10, ACM, New York, NY, USA, 2010, pp. 591-600. doi:10.1145/1772690.1772751. URL http://doi .acm.org/10.1145/1772690.1772751

[10] A. L. Traud, P. J. Mucha, M. A. Porter, Social structure of facebook networks, Physica A: Statistical 
Mechanics and its Applications $391 \quad$ (16) (2012) 4165-4180.

doi:10.1016/j.physa.2011.12.021.

URL http://dx.doi.org/10.1016/j.physa.2011.12.021

[11] R. Kumar, J. Novak, A. Tomkins, Structure and evolution of online social networks, in: Proceedings of the 12th ACM SIGKDD International Conference on Knowledge Discovery and Data Mining, KDD '06, ACM, New York, NY, USA, 2006, pp. 611-617. doi:10.1145/1150402.1150476. URL http://doi .acm.org/10.1145/1150402.1150476

[12] C. P.-Y. Chin, N. Evans, K.-K. R. Choo, Exploring factors influencing the use of enterprise social networks in multinational professional service firms, Journal of Organizational Computing and Electronic Commerce 25 (3) (2015) 289-315.

[13] C. P.-Y. Chin, N. Evans, K.-K. R. Choo, F. B. Tan, What influences employees to use enterprise social networks? a socio-technical perspective., in: PACIS, 2015, p. 54.

[14] C. P.-Y. Chin, K.-K. R. Choo, N. Evans, Enterprise social networks: a successful implementation within a telecommunication company.

[15] A. Heravi, D. Mani, K.-K. R. Choo, S. Mubarak, Making decisions about self-disclosure in online social networks, in: Proceedings of the 50th Hawaii International Conference on System Sciences, 2017.

[16] D. G. Rand, M. A. Nowak, J. H. Fowler, N. A. Christakis, Static network structure can stabilize human cooperation, Proceedings of the National Academy of Sciences 111 (48) (2014) 17093-17098.

[17] O. Yagan, D. Qian, J. Zhang, D. Cochran, Conjoining speeds up information diffusion in overlaying social-physical networks, IEEE Journal on Selected Areas in Communications 31 (6) (2013) 1038-1048.

[18] M. Dickison, S. Havlin, H. E. Stanley, Epidemics on interconnected networks, Physical Review E 85 (6) (2012) 066109. 
[19] J. Zhang, D. Brackbill, S. Yang, D. Centola, Efficacy and causal mechanism of an online social media intervention to increase physical activity: results of a randomized controlled trial, Preventive medicine reports 2 (2015) 651657.

[20] F. Radicchi, Driving interconnected networks to supercriticality, Physical Review X 4 (2) (2014) 021014.

[21] B. Parhami, Swapped interconnection networks: Topological, performance, and robustness attributes, Journal of Parallel and Distributed Computing 65 (11) (2005) 1443-1452.

[22] F. Radicchi, A. Arenas, Abrupt transition in the structural formation of interconnected networks, Nature Physics 9 (11) (2013) 717-720.

[23] F. Zhou, H. B.-L. Duh, M. Billinghurst, Trends in augmented reality tracking, interaction and display: A review of ten years of ismar, in: Proceedings of the 7th IEEE/ACM International Symposium on Mixed and Augmented Reality, ISMAR '08, IEEE Computer Society, Washington, DC, USA, 2008, pp. 193-202. doi:10.1109/ISMAR.2008.4637362.

URL http://dx.doi.org/10.1109/ISMAR .2008.4637362

[24] T. Olsson, M. Salo, Narratives of satisfying and unsatisfying experiences of current mobile augmented rea in: Proceedings of the SIGCHI Conference on Human Factors in Computing Systems, CHI '12, ACM, New York, NY, USA, 2012, pp. 2779-2788. doi: $10.1145 / 2207676.2208677$.

URL http://doi.acm.org/10.1145/2207676.2208677

[25] K. Rehrl, E. Häusler, S. Leitinger, D. Bell, Pedestrian navigation with augmented reality, voice and digital map: final results from an in situ field st Journal of Location Based Services 8 (2) (2014) 75-96. doi:10.1080/17489725.2014.946975

URL http://dx.doi .org/10.1080/17489725.2014.946975 
[26] T. Liao, L. Humphreys, Layar-ed places: Using mobile augmented reality to tactically reengage, reproduc New Media \& Society $17 \quad$ (9) (2014) 1418-1435. doi:10.1177/1461444814527734. URL http://dx.doi.org/10.1177/1461444814527734

[27] M. Billinghurst, S. Kim, A. Dunser, G. A. Lee, Cityviewar: A mobile outdoor ar application for city visualization, 2012 IEEE International Symposium on Mixed and Augmented Reality - Arts, Media, and Humanities (ISMAR-AMH) 00 (undefined) (2012) 57-64. doi:doi.ieeecomputersociety.org/10.1109/ISMAR-AMH. 2012.6483989.

[28] G. A. Lee, M. Billinghurst, A component based framework for mobile outdoor ar applications, in: Proceedings of the 12th ACM SIGGRAPH International Conference on Virtual-Reality Continuum and Its Applications in Industry, VRCAI '13, ACM, New York, NY, USA, 2013, pp. 207-210. doi:10.1145/2534329.2534344.

URL http://doi.acm.org/10.1145/2534329.2534344

[29] T. Althoff, R. W. White, E. Horvitz, Influence of pokmon go on physical activity: Study and implications (2016). arXiv:arXiv:1610.02085.

[30] J. Zhang, D. Brackbill, S. Yang, J. Becker, N. Herbert, D. Centola, Support or competition? how online social networks increase physical activity: A randomized controlled trial, Preventive Medicine Reports 4 (2016) 453-458.

[31] M. McCartney, Margaret mccartney: Game on for pokémon go, BMJ 354 (2016) i4306.

[32] A. Yuhuas, Pokemon go: armed robbers use mobile game to lure players into trap, The Guardian 11.

[33] L. Yangchen, Government will monitor impact of pokemon go on society: Yaacob, http://www.straitstimes.com/singapore/government-will-monitor-impact-of-pokemon-go-on-s accessed: 2016-11-22. 
[34] A. H. Catherine, A. I. Barbara, Social networks and social support, Health behavior and health education: Theory, research, and practice 4 (2008) 189-210. 
Tingmin Wu received the Bachelor of Information Technology degree (with first class Hons.) from Deakin University Australia in 2016. She is working toward the Ph.D. degree at the School of Information Technology, Deakin University. Her research interests include cyber security, especially in social spam detection.

Derek Wang received his Bachelor degree of Engineering from Huazhong University of Science and Technology (HUST), China (2011); M.Sc. by research degree from Deakin University, Australia (2016). Currently, Derek is a PhD student with Deakin University and CSIRO Data61, Australia. His research interests include machine learning, advanced deep neural network, and application of deep learning in complex network, social networking security, distributed system security, and network risk assessment.

Dr. Sheng Wen received the degree in computer science from the Central South University of China in 2012, and the Ph.D. degree from the School of Information Technology, Deakin University, Australia, in 2015. Since then, he has been a Lecturer with Deakin University. His focus is on modeling of virus spread, information dissemination, and defense strategies for the Internet threats. $\mathrm{He}$ is also interested in the techniques of identifying information sources in networks.

Prof. Yang Xiang received his PhD in Computer Science from Deakin University, Australia. He is the Director of Centre for Cyber Security Research, Deakin University. His research interests include network and system security, data analytics, distributed systems, and networking. In particular, he is currently leading his team developing active defense systems against large-scale distributed network attacks. He is the Chief Investigator of several projects in network and system security, funded by the Australian Research Council (ARC). He has published more than 200 research papers in many international journals and conferences. Prof Yang is a Senior Member of the IEEE.

Prof. Wanlei Zhou received the B.Eng and M.Eng degrees from Harbin Institute of Technology, Harbin, China in 1982 and 1984, respectively, and the PhD degree from The Australian National University, Canberra, Australia, in 1991, all in Computer Science and Engineering. He also received a DSc degree (a higher Doctorate degree) from Deakin University in 2002. He is currently the Alfred Deakin Professor (the highest honour the University can bestow on a member of academic staff), Chair of Information Technology, and Associate Dean (International Research Engagement) of Faculty of Science, Engineering and Built Environment, Deakin University. Professor Zhou has been the Head of School of Information Technology twice (Jan 2002-Apr 2006 and Jan 2009-Jan 2015) and Associate Dean of Faculty of Science and Technology in Deakin University (May 2006-Dec 2008). His research interests include distributed systems, network security, bioinformatics, and e-learning. Professor Zhou has published more than 300 papers in refereed international journals and refereed international conferences proceedings. Prof Zhou is a Senior Member of the IEEE.

Prof. Houcine Hassan is associate Professor of the Department of Computer Engineering in the Polytechnic University of Valencia, Spain, since 1994. He received a MS and PhD. degree in Computer Engineering from the Polytechnic University of Valencia, in 1993 and 2001 respectively. He joined the Industrial Informatics group in 1993, where he is participating in several research projects. His research interests covers a number of aspects of the development of hardware and software architectures including real-time systems support, embedded systems, hardware/software co-design, ubiquitous computing, Al agent based systems, autonomous systems, sensor networks, robotic architectures, behaviour and emotional systems, application and scheduling integration, QoS. 
Dr. Abdulhameed Al-Eliwi got his Ph.D. in Software Engineering, College of Engineering, Florida Institute of Technology-Melbourne, USA, 2002. He got his M.Sc. in Computer Science (Information Systems), College of Engineering, Florida Institute of Technology-Melbourne, USA, 1998and M ,.Sc. in Engineering Management (Systems Engineering), College of Engineering, Florida Institute of Technology-Melbourne, USA, 2003. His B.Sc. was also in Computer Information Systems with Honor, College of Computer and Information Science, KSU, Saudi Arabia, Riyadh 1992 


\section{Highlights}

In this paper, we study the formation, symbiosis and impact of two networks, which are the virtual social network of in-game characters, and the social network of players in the physical world. 


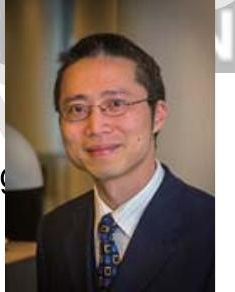




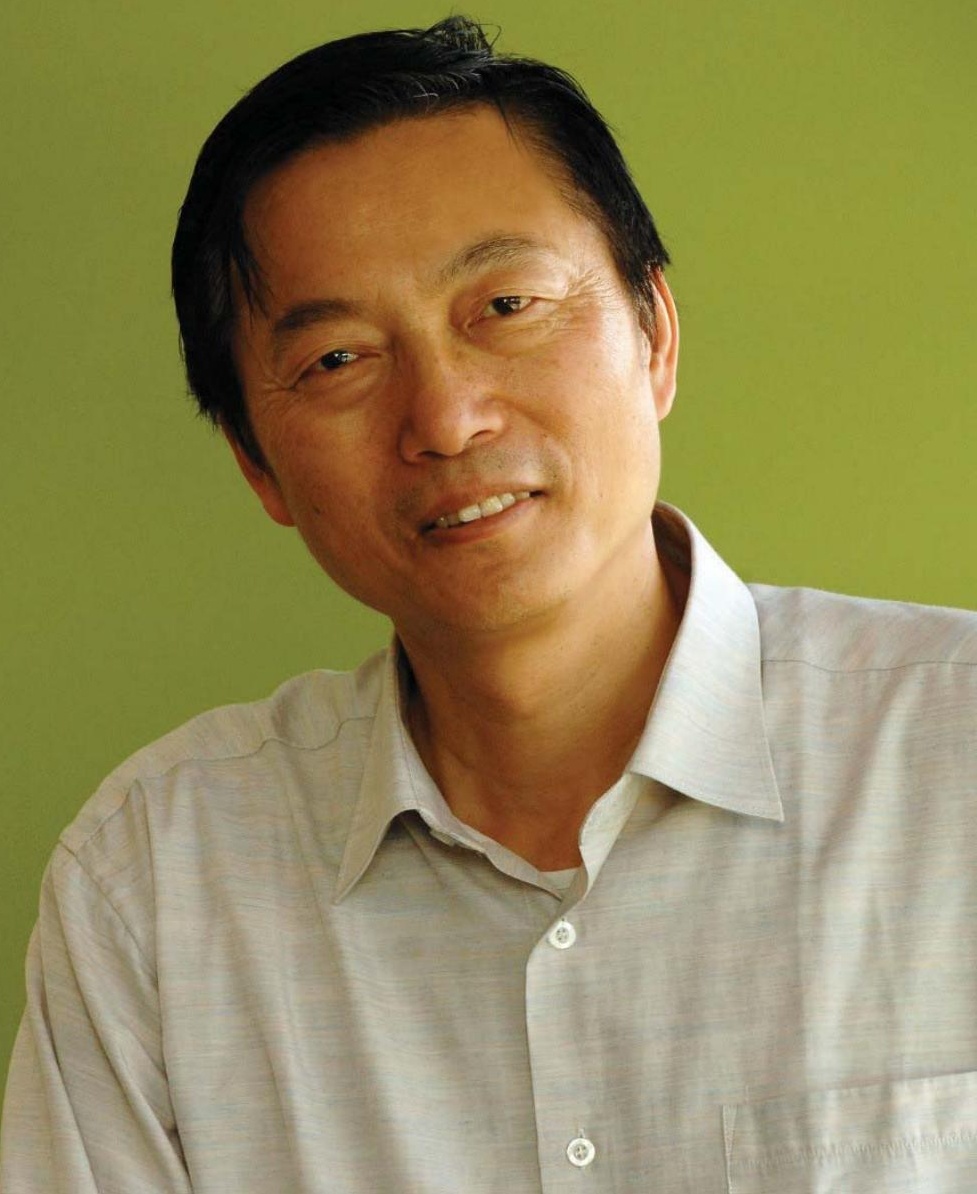




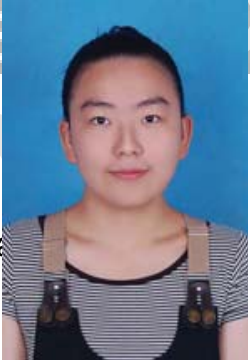




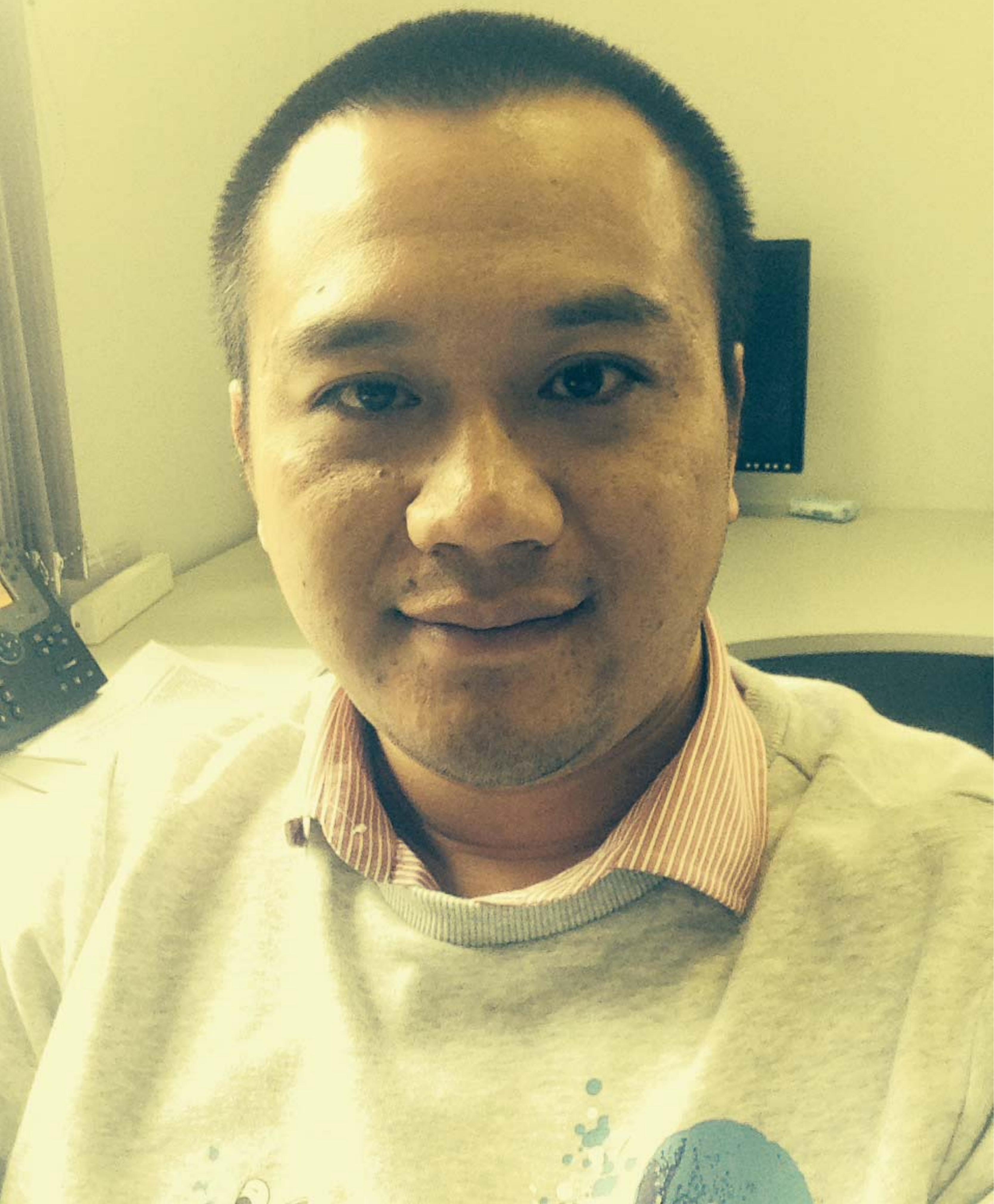




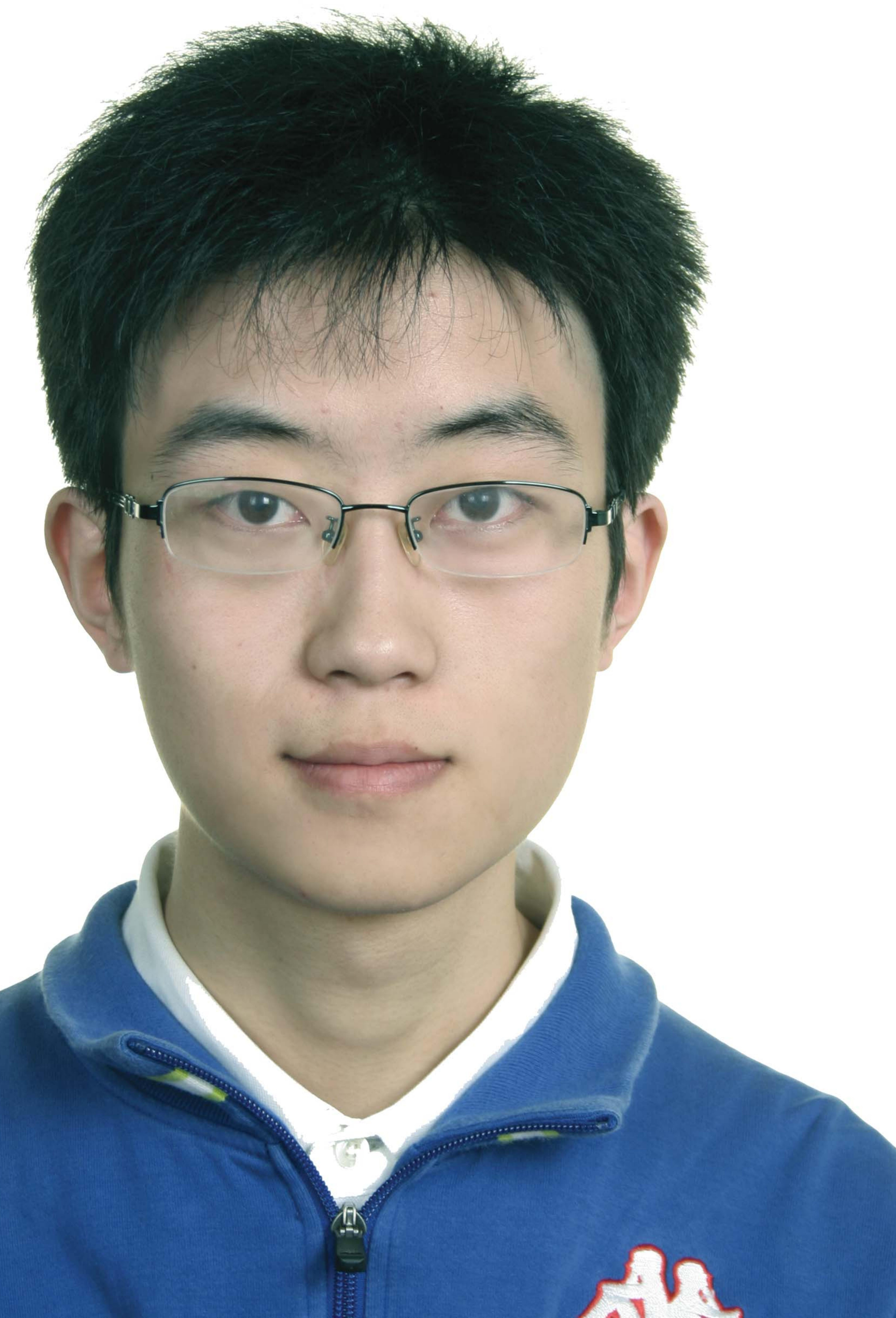




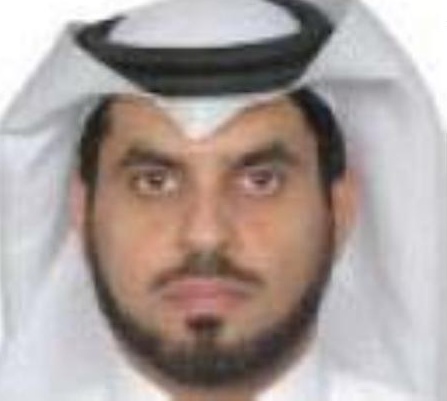

